\title{
Kluyvera ascorbata
}

National Cancer Institute

\section{Source}

National Cancer Institute. Kluyvera ascorbata. NCI Thesaurus. Code C86462.

A species of facultatively anaerobic, Gram negative, rod shaped bacteria assigned to the phylum Proteobacteria. This species is motile by peritrichous flagella, catalase and indole positive, oxidase negative, positive for lysine decarboxylase, and can grow on cefsulodin irgasan novobiocin agar in culture. K. ascorbata is rarely isolated from humans and tends to be pathogenic only in immunocompromised individuals. 